\title{
The combined ring rolling process and the stiffness condition
}

\author{
Jian Lan ${ }^{1,2, a}$, Wei Zhang ${ }^{3}$, Lin Hua ${ }^{1,2}$, and Huajie $\mathrm{Mao}^{3}$ \\ ${ }^{1}$ Hubei Key Laboratory of Advanced Technology for Automotive Components, WHUT, \\ Wuhan 430070, China \\ ${ }^{2}$ Hubei Collaborative Innovation Center for Automotive Components Technology, WHUT, \\ Wuhan 430070, China \\ ${ }^{3}$ School of Materials Science and Engineering, Wuhan University of Technology, \\ Wuhan 430070, China
}

\begin{abstract}
Different from the conventional ring rolling mill, the combined ring rolling mill has not only one main roll and one mandrel to enlarge the ring blank, but also two assistant rolls to deform the ring blank with the main roll continuous feeding. The novel ring rolling process can have three working modes: 1 ) conventional ring rolling; 2) cross rolling with three rolls surrounding the ring blank; 3) conventional ring rolling with cross rolling. Moreover, the new ring rolling process allows the transferring from one working mode to another. The process and the mill provide more flexibility for manufacturing different ring products. The stiffness condition at different working modes and the smoothly transferring conditions are first studied carefully. Then the combined ring rolling model for deep groove ring is established and evaluated.
\end{abstract}

\section{Introduction}

Ring rolling is an incremental bulk metal forming process to manufacture seamless rings with various diameters and cross sections. These rings are commonly used in bearing, aircraft, energy and automotive industry. The most important advantages of the ring rolling process are the favorable grain flow, smooth surface and better mechanical properties of rings, and considerable savings in man-hours and material needed. Because of the world energy crisis, the sustainable development required the metal forming industry to integrate as many operations as possible into one process. This is very inviting suggestion for hot ring rolling to combine two or more operations into one, which can reduce the heating times between operations. Usually, the ring blank experiences the upsetting, piercing and preforming ideally before ring rolling process. After preforming, the temperature of ring blank often drops below the austenitizing temperature, and the ring blank is not suitable for conventional ring rolling process and need to be heated again. Another scenario is that the rolled ring with roundness error needs to be heated and corrected by cross rolling with three rollers in practice. The cross rolling with three rollers is applied to produce the pie shape with round circumference (diameter $>$ height). In this paper, the combined ring rolling process is proposed to take the advantages of the ring rolling and cross rolling with three rollers, to shorten the manufacturing chain for the profile ring products.

\footnotetext{
${ }^{\text {a }}$ Corresponding author: jlan@whut.edu.cn
}

This is an Open Access article distributed under the terms of the Creative Commons Attribution License 4.0, which permits unrestricted use, distribution, and reproduction in any medium, provided the original work is properly cited. 


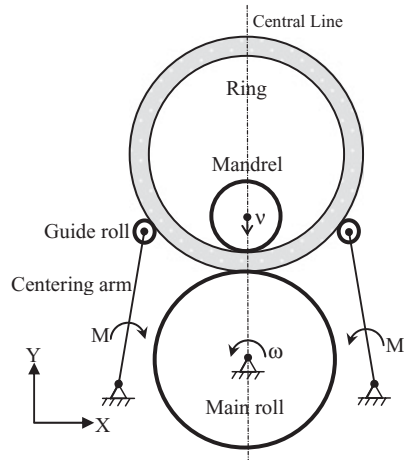

Figure 1. Schematic diagram of ring rolling mill.

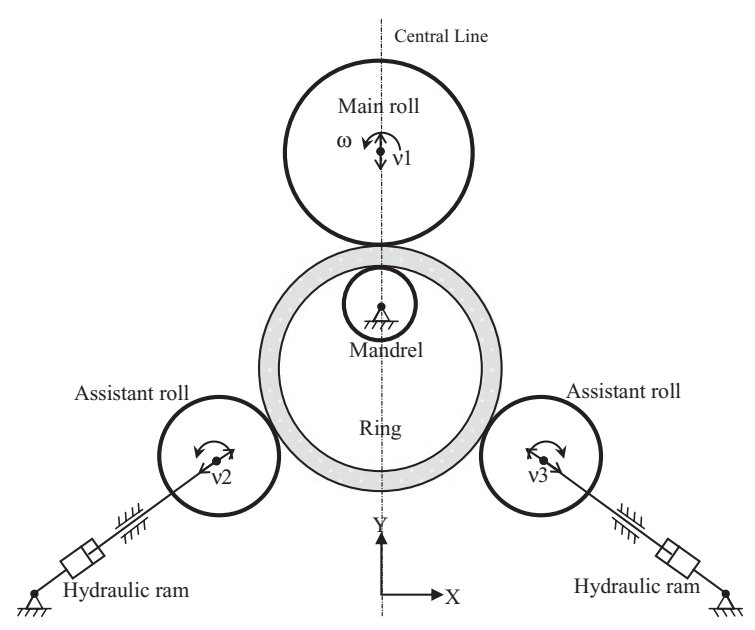

Figure 2. Schematic diagram of combined ring rolling mill.

Many researches focused on to accelerate the simulation of the process. Joun [1] applied an axisymmetric forging approach to optimize the preform design. Forouzan [2, 3] proposed the thermal spokes method to simulate the guide roll in the finite element analysis of ring rolling, and to avoid the contact boundary condition. Davey $[4,5]$ employed two meshes and ALE flow formulation to focus on deformation in the biting area and speed up the simulation. But there are geometry and material nonlinearities in the ring rolling process, which are obstacles to simulate the ring rolling process stably and quickly especially in the process design stage. And from the process design engineers' point of view, the range of design parameters is more favorable and more convenient to use. Hua [6] provided the extremum parameters for the ring rolling process. In this paper, the stiffness condition is proposed to estimate the limit parameters for the combined ring rolling process.

\section{Combined ring rolling mill}

As shown in Fig. 1, the ring rolling process is non-steady state throughout and the work-piece is endless with a curvature and its radius increases continuously. The ring reduction per pass is small and the final dimensions are obtained through continuous multi-pass rolling. The guide rolls support the ring in the central line by the centering arms, while the main roll position is fixed and mandrel is approaching to the main roll. The proposed combined ring rolling, as shown in Fig. 2, is different from the ring rolling, which has the mandrel position fixed and main roll can move up and down, $v 1$. Moreover, there are two assistant rolls symmetrically allocated to the central line. The main roll and the assistant rolls are at $120^{\circ}$ angle to each other and can be controlled independently. The only torque, which keeps the ring rolling, is still provided by the main roll in the rolling process. The rolling force is also provided by the main roll. The two assistant rolls can move along the symmetrical tracks, ( $v 2$ and $v 3$ ), and can provide the supporting force with main roll by the hydraulic rams as shown in Fig. 2. Also, the positions of the assistant rolls can be fixed by adjusting the hydraulic control system during rolling process.

\section{Working modes}

Based on the description of the combined ring rolling mill, it could have some possible work modes, as shown in Fig. 3. First of all, the basic mode is the cross rolling with three rolls as shown in the Fig. 3a,c 


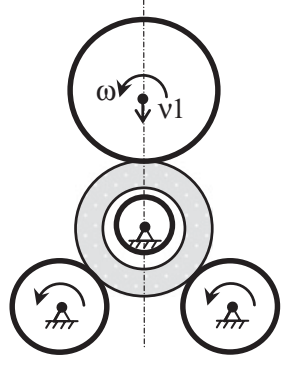

(a) roundness regularizing

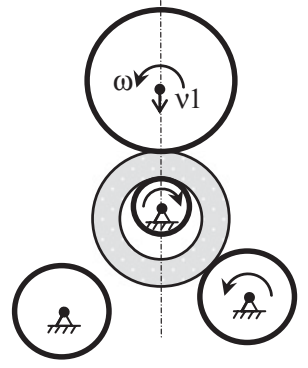

(d) ring rolling

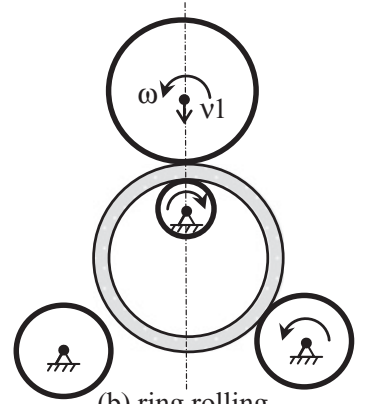

(b) ring rolling

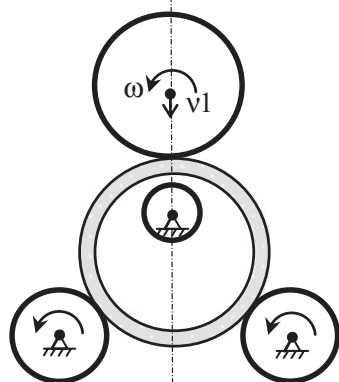

(e) outer profile filling

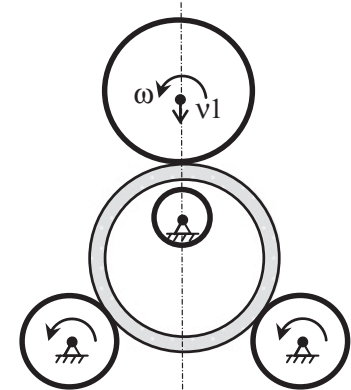

(c) outer profile filling

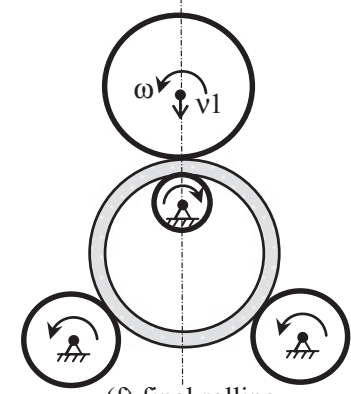

(f) final rolling

Figure 3. Possible working modes of combined ring rolling mill.

and e. In this mode, the ring workpiece contact with the main roll and two assistant rolls and without the mandrel. The ring workpiece is rotated by the main roll and outer profile of the ring could be deformed. The ring will not enlarge, because the mandrel and main roll do not make up the biting area like the ring rolling process. In this mode, the ring workpiece could be regularized to roundness and the outer profile. The second mode is the traditional ring rolling as shown in the Fig. $3 \mathrm{~b}$ and $\mathrm{d}$. In this mode, the ring workpiece can be enlarged by reducing the thickness of the ring. The third mode is the combined mode as shown in Fig. 3f. In this mode, the ring is bit by the main roll and mandrel, while is supported by main roll and two assistant rolls. So the ring can enlarge and regularize the outer contour, depending on the contact situation in the biting area.

The combined ring rolling mill provides these modes and enrich the rolling process, possibly provide shorten manufacturing chain for ring products with outer profile. Here, two combined processes are highlighted. The first combined process A has 3 stages, as shown in Fig. 3, $a \rightarrow b \rightarrow c \rightarrow$. At the stage 1, the ring blank, which could have relative large dimension error, can be regularized. At the stage 2, the ring blank can be rolled to enlarge the diameter and reduce the thickness. At the stage 3 , the ring workpiece can be regularized again. The second combined process B has 3 stages too, as shown in Fig. 3, $\mathrm{d} \rightarrow \mathrm{e} \rightarrow \mathrm{f}$. At the stage 1 , the ring blank is enlarged by the ring rolling mode. At the stage 2, the diameter of the ring reaches the design value, and the outer profile of the ring is formed by cross rolling. At the stage 3, the inner surface of the ring is formed by the combined mode.

\section{Ring stiffness and examples}

The simplified models for mechanical analysis are list in the Fig. 4. The maximum bending moment is $M=\frac{2 \sqrt{3} \pi+9}{12 \pi} F R_{a}$ for the ring rolling, and $M=\left(-\frac{\sqrt{3}}{6} \frac{3}{2 \pi}\right) F R_{a}$ for cross rolling, where the $R_{a}$ is average of the outer and inner diameter of the ring, as shown in Fig. 5 and Table 1, F is the rolling force, which can be estimated according to Hua [7]. Then the thickness of ring h, can be used as the criterion 


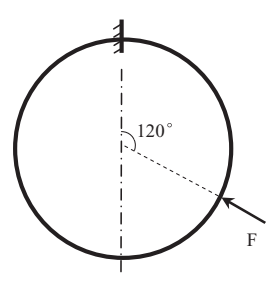

(a) ring rolling

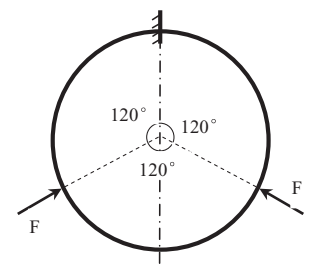

(b) cross rolling

Figure 4. Kinetic diagrams of different working modes of combined ring rolling.

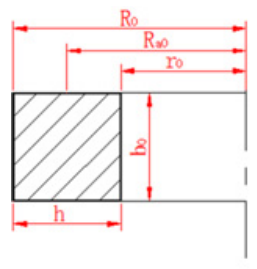

(a)

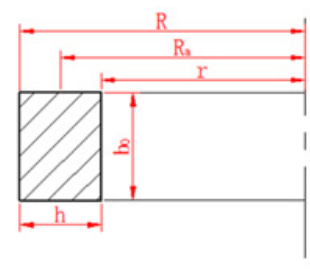

(b)

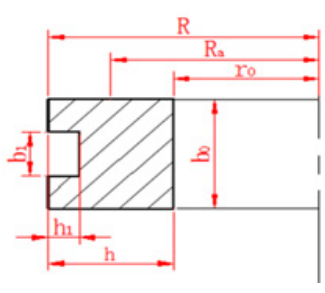

(c)

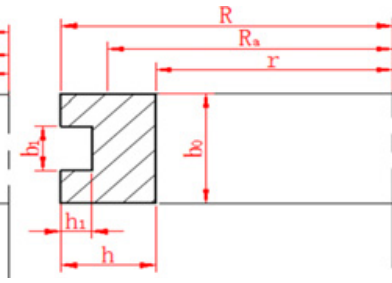

(d)

Figure 5. The ring blank (a), interim ring by ring rolling (b), interim ring by cross rolling (c), and the designed ring product (d).

Table 1. The dimensions of ring product and ring blank.

\begin{tabular}{ccccccccc}
\hline \multicolumn{4}{c}{ Ring product (mm) } & \multicolumn{1}{c}{ Ring blank (mm) } \\
\hline $\mathrm{R}$ & $\mathrm{r}$ & $R_{a}$ & $h_{1}$ & $b_{1}$ & $b_{0}$ & $R_{0}$ & $r_{0}$ & $R_{a 0}$ \\
350 & 190 & 270 & 85 & 90 & 285 & 284.46 & 105 & 194.73 \\
\hline
\end{tabular}

Table 2. Parameters of the combined ring rolling mill.

\begin{tabular}{rlrl}
\hline Radius of main roll & $390(\mathrm{~mm})$ & Max feeding rate & $7.26(\mathrm{~mm} / \mathrm{r})$ \\
Radius of asst. roll & $200(\mathrm{~mm})$ & Min feeding rate & $0.0217(\mathrm{~mm} / \mathrm{r})$ \\
Radius of mandrel & $50(\mathrm{~mm})$ & Strength of ring material & $90(\mathrm{MPa})$ \\
\hline
\end{tabular}

for the stiffness condition, by the bending theory and material strength. The feeding rate is key factor to determine the rolling force and the criterion of stiffness condition. There are max and min feeding rate listed in Table 2. Correspondingly, the criterion for max and min feeding rates are hcmax and hcmin as shown in Fig. 6. The thickness of ring h being larger than the criterion means the ring stiffness is enough to keep on rolling process. Otherwise, the ring will have less stiffness and be collapsed by rolls.

Two combined processes mentioned above are illustrated below, and the ring blank, product and notations are shown in Fig. 5 and Table 1. The parameters of the combined ring rolling mill are list in Table 2.

For the combined process A, the ring blank, as shown in Fig. 5a, will firstly be deformed to the interim ring as shown in Fig. 5c with unchanged inner diameter, and then to the final shape as shown in Fig. 5d. At the stage 1, with the groove h1 deepening, the thickness h increases and is larger than hcmax and hcmin, as shown in Fig. 6a. This means the cross rolling is stable for the ring blank. At the stage 2, with the average radius of the ring Ra increasing, the thickness $h$ decreases and is smaller than hcmax at 239 and homin at 203. This means the ring rolling can not be carried out. 


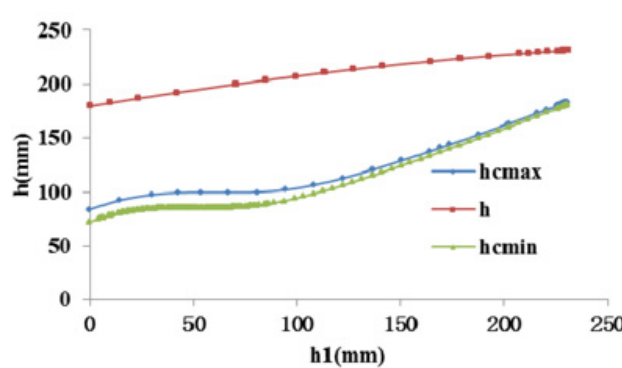

(a)

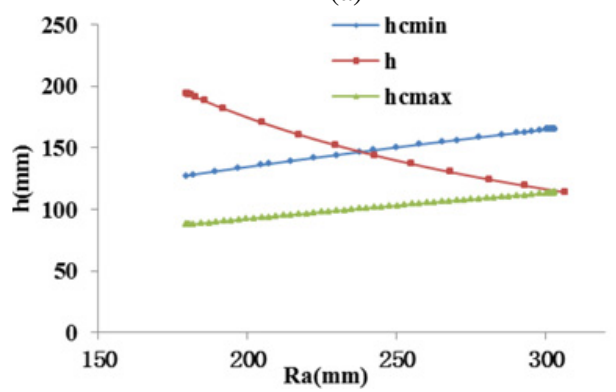

(c)

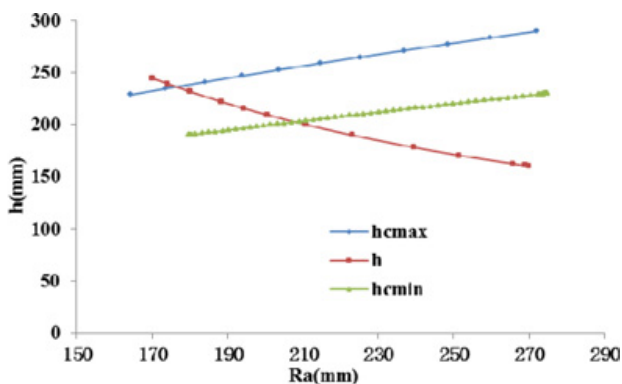

(b)

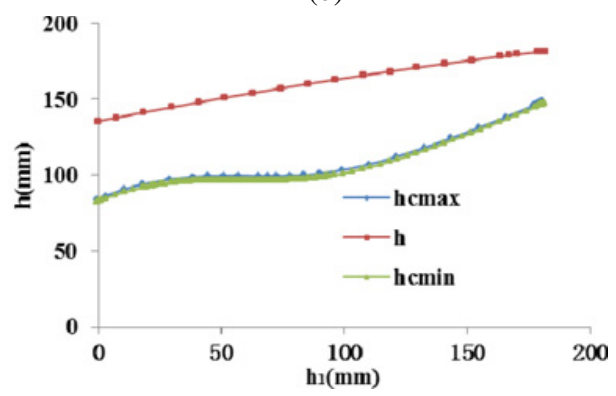

(d)

Figure 6. The ring stiffness condition in Process A stage1 (a), Process A stage 2 (b), Process B stage 1 (c), and Process B stage 2 (d).

For the combined process B, the ring blank will firstly be deformed to the interim ring as shown in Fig. 5b with small groove (omitted here), and then to the final shape. At the stage 1, with the average radius of the ring increasing, the thickness $\mathrm{h}$ decreases and is smaller than hcmax at 147 and hcmin 113. This means that the ring diameter could enlarge to 300 if selecting appropriate feeding rate. At the stage 2, with the groove h1 deepening, the thickness $\mathrm{h}$ increase and is larger than hcmax and hcmin. This means the cross rolling is stable for the rolled ring.

Comparing process A and $\mathrm{B}$, the combined process $\mathrm{B}$ is more suitable to be applied in practice. The experiment of the combined ring rolling has been carried out and confirms the analysis above. The mill used is with fixed assistant rolls during rolling process.

\section{Conclusions}

The combined ring rolling allows the transferring from one working mode to another. The combined process B has more application prospect.

\section{References}

[1] M.S. Joun, J.H. Chung, R. Shivpuri, An axisymmetric forging approach to perform design in ring rolling using a rigid-viscoplastic finite element method, Int J of Mach Tools \& Manu., 38 (1998)

[2] M.R. Forouzan, M. Salimi, M.S. Gadala, Three-dimensional FE analysis of ring rolling by employing thermal spokes method, Int J of Mechanical Sciences, 45 (2003)

[3] M.R. Forouzan, M. Salimi, M.S. Gadala, Guide roll simulation in FE analysis of ring rolling, Journal of Materials Processing Technology, 142, 1 (2003) 


\section{MATEC Web of Conferences}

[4] K. Davey, M.J. Ward, A practical method for finite element ring rolling simulation using the ALE flow formulation, International Journal of Mechanical Sciences, 44 (2002)

[5] K. Davey, M.J. Ward, The practicalities of ring rolling simulation for profiled rings, Journal of Materials Processing Technology, 125-126 (2002)

[6] L. Hua, Z. Zhao, The extremum parameters in ring rolling, Journal of Materials Processing Technology, 69 (1997)

[7] L. Hua, X. Huang, C. Zhu, Theory and Technology of Ring Rolling (China Machine Press, Beijing, 2001) 